\title{
Zur Geschichte des Äthers im 18. Jahrhundert. George-Louis Lesages System der corpuscules ultramondains
}

\author{
Jutta Berger $\uparrow$
}

\section{Summary}

The article presents an account of Lesage's theory of corpuscules ultramondains and ether. It shows that essential attributes of the antique concept of ether still belong to his speculative physics. In accordance with this concept, Lesage understood the corpuscules ultramondains as subtle celestial matter and carriers of the primordial movements in the universe which were characterised by their proximity to the divine. On the other hand, Lesage was engaged in the contemporary debate concerning the problem of causa gravitatis. His position turns out to be halfway between dynamic corpuscularity

* Frau Dr. Jutta Berger $(* 2.10 .1960, \uparrow 12.2 .2005)$ verstarb - kurz nachdem sie ihren Artikel eingereicht hatte - an den Folgen einer langen Krankheit. - Nach einem Studium der Chemie an der Johann Wolfgang Goethe-Universität Frankfurt und der Wissenschaftsgeschichte an der Technischen Universität Berlin hatte sie ihre Dissertation der Geschichte der Chemie im 19. Jahrhundert gewidmet (publiziert unter dem Titel Affinität und Reaktion. Über die Entstehung der Reaktionskinetik in der Chemie des 19. Jahrhunderts, Studien und Quellen der Chemie, Bd. 11, Verlag für Wissenschafts- und Regionalgeschichte, Berlin 2000). In ihrer Forschung beschäftigte sie sich mit der Geschichte des Verhältnisses von Naturphilosophie und gesellschaftlicher Moral bei Paracelsus, den Kosmogonien der Philosophen-Chemiker im 16. und 17. Jahrhundert und allgemeiner mit der Geschichte der Chemie und Physik in der Frühen Neuzeit. Sie hat dazu verschiedene Arbeiten in Fachzeitschriften und Sammelbänden (Acta historica Leopoldina, Centaurus, Annals of Science usw.) publiziert. Jutta Berger erhielt Stipendien von der Chemical Heritage Foundation, vom Max-Planck-Institut für Wissenschaftsgeschichte Berlin und von der Herzog August Bibliothek Wolfenbüttel und war zuletzt als Mitarbeiterin der Universität der Bundeswehr München an einem Forschungsprojekt über die Geschichte des Äthers tätig. (V. B.)

** Der Artikel präsentiert ein Teilergebnis eines dreijährigen DFG-Forschungsprojekts zur Geschichte des Äthers und der aktiven Prinzipien, das an der Universität der Bundeswehr München durchgeführt wurde. Mein besonderer Dank gilt dem Projektleiter Herrn Prof. Ivo Schneider für anregende Diskussionen und weiterführende Hinweise und meinen Freunden und Kollegen PD Dr. Michaela Boenke und Jörg Sacher für kritische Lektüre und hilfreiche Kommentare. Nicht zuletzt ein herzliches Dankeschön an Herrn Prof. Andreas Kleinert für die Überlassung des Manuskripts seiner Edition der Euler-Lesage-Korrespondenz. 
and mechanistic physics. With the former he shared the rejection of vortex theories and the adherence to the corpuscular theory of light and the nutshell theory, with the latter the rejection of action at-a-distance and forces inherent to matter. Contrary to the accusation that he plagiarised Fatio de Duillier's theory of gravitation, it is shown that Lesage formulated a gravitational mechanism essentially different to Fatio's.

Lesage used the term ether for the theory of chemical affinities. His attempt to reduce them to gravitational force failed. The complexity of chemical phenomena could not be mastered theoretically by reduction to a uniform natural force.

Keywords: history of ether; George-Louis Lesage; theory of active principles; history of mechanistic physics in the 18th century

\section{Zusammenfassung}

Der Artikel gibt eine Darstellung von Lesages Theorien der corpuscules ultramondains und des Äthers. Er zeigt, dass wesentliche Attribute des antiken Ätherbegriffs in seine spekulative Physik eingingen. Als subtile Himmelsmaterie, als Träger der Urbewegungen im Kosmos und ausgezeichnet durch ihre Nähe zum Göttlichen, blieben die corpuscules ultramondains dem antiken Ätherbegriff verpflichtet. Andererseits stand Lesage mitten in der aktuellen Auseinandersetzung um das Problem der causa gravitatis. Seine Position wird als eine mittlere zwischen dem dynamischen Korpuskularismus und der mechanistischen Physik identifiziert. Mit ersterem verband ihn die Ablehnung der Wirbeltheorie, das Bekenntnis zur Korpuskulartheorie des Lichts und zur Nut-shell-Theorie, mit letzterer die Ablehnung materieinhärenter Kräfte und Fernwirkungen. Hinsichtlich des Plagiatsvorwurfs gegenüber Fatio de Duillier wird nachgewiesen, dass Lesages Gravitationstheorie im Kern einen anderen Mechanismus vorsah als diejenige Fatios.

Lesage verwendete den Terminus Äther im Rahmen der Theorie chemischer Affinitäten. Sein Versuch, sie auf die Gravitationskraft zurückzuführen, scheiterte. Die Komplexität chemischer Phänomene liess sich durch Reduktion auf eine uniforme Naturkraft theoretisch nicht bewältigen.

\section{Einleitung}

Die Geschichte des Äthers ist alt und komplex und reicht weit zurück in die Philosophie der Vorsokratik, und doch, so scheint es, lassen sich über die 
Differenzen der verschiedenen philosophischen Schulen hinweg Merkmale bestimmen, die den Äther von jeher charakterisierten. «Unentschieden auf der Schwelle von Immateriellem und Materiellem» ${ }^{1}$ stehend, galt der Äther immer als eine besondere Substanz. Er war Licht, Seele und Himmelsstoff, ausgezeichnet durch seine Nähe zum Göttlichen und in seiner Subtilität verschieden von den anderen Elementen. Als Himmelsstoff kam ihm die Funktion der Raumerfüllung zu. Als Vermittler zwischen der göttlichen Kraft und der passiv gedachten groben Materie war der Äther Träger der Urbewegungen im Kosmos².

In der stark von mythischem Denken geprägten pseudoplatonischen Schrift Epinomis wurde die Vorstellung von fünf Grundkörpern formuliert, aus denen alle Naturdinge entstanden sein sollten: Feuer, Wasser, Luft, Erde und Äther. Aus Ätherstoff waren Dämonen und Luftwesen gebildet, denen eine mittlere und vermittelnde Position zwischen Menschen und Göttern zukam $^{3}$. In der aristotelischen Philosophie wurde die Differenz zu den gewöhnlichen Elementen strikter bestimmt. Als unveränderlicher und unvergänglicher Körper war der Äther nicht wie die anderen Elemente durch Qualitäten ausgezeichnet, die in Gegensatzpaaren gefasst werden konnten, sondern durch seine ewige Bewegung, die ihrerseits als eine kreisförmige gedacht wurde ${ }^{4}$. In der unechten Aristoteles-Schrift Über die Welt bildete der Äther den Ausgangsort der kosmischen Bewegung, das erste Glied einer Himmelsmechanik. «So nun leitet auch die Gottheit durch einfachen Anstoß des ersten Bereiches ihre Kraft an die nächsten Regionen weiter.» ${ }^{5}$

Der spirituelle Materialismus der Stoa identifizierte Äther und Feuer und erblickte in ihm die schöpferische Urkraft der Welt, die Gottheit selbst. So war für Zenon der Äther «der höchste Gott, der Träger des Geistes, von dem alles gelenkt wird $»^{6}$. Als das «reinste Feuer» bildete er den Himmel und durch seine kreisförmige Bewegung die Grenze der Weltkugel ${ }^{7}$. Der antike Atomismus stand der traditionellen Elementenlehre naturgemäss skeptisch gegenüber ${ }^{8}$. Doch auch der im 18. Jahrhundert vielgelesene und für Lesage, wie im folgenden deutlich werden soll, wichtige Autor Lukrez bediente sich des Ätherbegriffs. Der Äther war auch für ihn der Himmelsstoff, heiss und flammend, als «Bringer des Feuers» nicht klar von diesem geschieden, be-

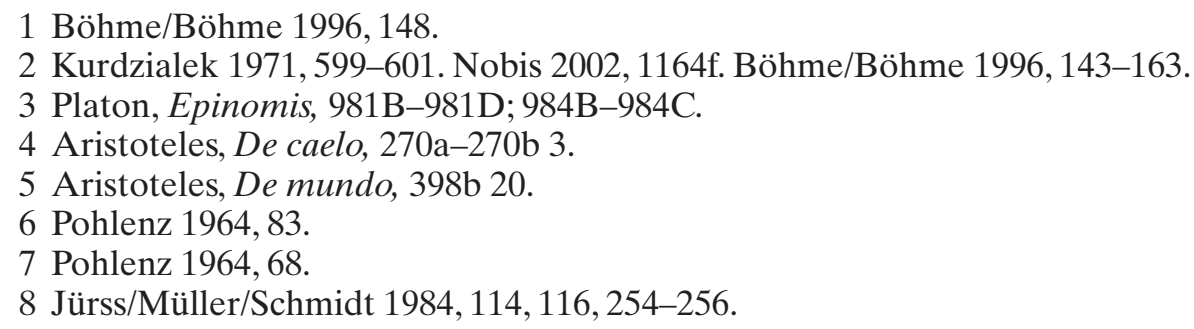


stehend aus kleinen, glatten und rundlichen Atomen. Ferner hatte Lukrez den Äther mit Expansivkraft ausgestattet: ein sich leicht verströmender Stoff, der sich im All in alle Richtungen ausdehnte .

In der frühneuzeitlichen Physik wurde der Ätherbegriff verwissenschaftlicht und im Rahmen konkreter physikalischer Fragestellungen zu einer Hypothese umgeformt. Newtons Gesetze hatten die vis gravitatis beschrieben, die Frage nach der causa gravitatis jedoch offengelassen. In dem Bestreben, die Gefahr rational nicht verstehbarer Fernwirkungen abzuwehren, kam nun wieder der Äther ins Spiel. Versehen mit seinen traditionellen Merkmalen, wenn auch korpuskulartheoretisch uminterpretiert, sollte der Äther der mechanistisch orientierten Physik zur Lösung des Problems der Kraftübermittlung in die Ferne verhelfen. Neben dem Gravitationsphänomen stellte die Lichtausbreitung einen weiteren Ort der Bewährung physikalischer Äthertheorien dar.

\section{Biographischer Hintergrund}

George-Louis Lesages (1724-1803) Physik steht in diesem Kontext. Seine Theorie der Himmelsmaterie versuchte eine Antwort auf die Frage nach der causa gravitatis und - hiervon abgeleitet - auf die der Übertragungsmechanismen anderer physikalischer und chemischer Kräfte. Sie bildet einen wichtigen Bestandteil der im 18. Jahrhundert intensiv geführten Auseinandersetzung um eine mechanistische Gravitationstheorie, zu der unter anderen Huygens, Leibniz und Euler vieldiskutierte Vorschläge eingebracht hatten ${ }^{10}$ und die darüber hinaus das besondere Interesse der gelehrten Welt Genfs, der Heimatstadt Lesages, fand ${ }^{11}$.

Folgt man der Darstellung seines Schülers Pierre Prevost (1751-1839), dann setzte zu Beginn des 19. Jahrhunderts eine gewisse Ermüdung gegenüber Versuchen zur Formulierung mechanistischer Gravitationstheorien $e{ }^{12}$. Dennoch umfing in den folgenden Jahrzehnten Lesage der Nimbus des genialen Aussenseiters. So erlebte seine Theorie in den siebziger Jahren des 19. Jahrhunderts eine erste Renaissance, als sie im Zusammenhang mit der Entwicklung der mechanischen Wärmetheorie zuerst von Rudolph Clausius und anschliessend von den britischen Physikern S. Tolver Preston

9 Lukrez, De natura rerum, II 1061-1066; V 440-490.

10 Isenkrahe 1881, 1-19.

11 Weiss 1988, 108-148.

12 Prevost 1818, Preface, X-XII. 
und Lord Kelvin aufgegriffen wurde ${ }^{13}$. Ein Jahrhundert später pries der Wissenschaftstheoretiker Laurens Laudan Lesage als Protagonisten einer modernen Methodologie der Physik, basierend auf dem Wechselspiel von Hypothese und Verifikation ${ }^{14}$. Auch in jüngster Zeit äusserten wieder Physiker Interesse an seiner Gravitationstheorie als bemerkenswertem Versuch, über rein mathematische Beschreibungen, wie sie Newton und Einstein geliefert hätten, hinausgehend die «eigentliche Ursache» der Gravitation zu erfassen ${ }^{15}$.

Lesage selbst hat zeitlebens nur wenig publiziert. Prevosts Biographie gibt eine einfühlsame Schilderung der Kindheit seines Lehrers. Seine Beschreibung der freudlosen Atmosphäre in Lesages Elternhaus und einer repressiven Erziehung lässt die Gründe für diese publizistische Zurückhaltung erahnen ${ }^{16}$. So lernte Lesage früh, dass es bei weitem das günstigste ist, eigene Gefühle und Gedanken für sich zu behalten, um schmerzhafte Konflikte zu vermeiden. Die harte Haltung seines Vaters förderte in ihm einen Hang zu Schüchternheit und Einsamkeit, der ihm später von grossem Nachteil war. So sah er sich ausserstande, das Bewerbungsverfahren um eine Professur für Mathematik, die um 1750 in Genf zu besetzen war, psychisch durchzustehen $^{17}$. Nach einem Studium am Auditoire de Philosophie, der Genfer Akademie, absolvierte er auf Drängen seines Vaters ein Medizinstudium in Paris und in Basel - wo er jedoch Daniel Bernoullis (1700-1782) Vorlesungen über Wärmetheorie der ungeliebten Medizin bei weitem vorzog. Dem väterlichen Vorbild folgend, liess er sich mehr oder weniger notgedrungen als Privatlehrer in Genf nieder. Die Eröffnung einer Arztpraxis, die eigentlich angestrebt worden war, war ihm aus juristischen Gründen wegen fehlender Vollbürgerschaft verboten.

Im 18. Jahrhundert boten aufgrund seiner publizistischen Abstinenz im wesentlichen die 1758 von der Académie de Rouen preisgekrönte Schrift Essai de Chymie méchanique, die 1761 in überarbeiteter und ergänzter Form gedruckt wurde ${ }^{18}$, und der 1784 in den Berliner Nouveaux Mémoires de l'Académie Royale des Sciences et Belles-Lettres erschienene Artikel Lucrèce Neutonien Zugang zu seiner Theorie ${ }^{19}$. Doch führte Lesage als wohlbekanntes Mitglied der République des Lettres eine umfangreiche Korrespondenz mit namhaften Kollegen wie D'Alembert, Bonnet, Boscovich, Clairaut, Euler

13 Stosz 1884, 4f. Aronson 1964, 59f. (der Clausius’ Anteil unterschlägt).

14 Laudan 1974, 241-252; Laudan 1981, 164-169.

15 Edwards 2002, Preface.

16 Prevost $1805,1-140$.

17 Prevost 1805,70 .

18 Evans 2002, 39.

19 Verzeichnis der Publikationen Lesages vgl. Weiss 1988, 439f. Ein Verzeichnis der Handschriften Lesages in der Genfer Bibliothèque Publique et Universitaire gibt Stosz 1884, 59f. 
und Lambert, in der er seine Ideen zur Diskussion stellte. Ausschnitte aus diesen Briefen hat Prevost posthum im Rahmen der Notice de la vie et des écrits de George-Louis Lesage veröffentlicht. Die für heutige Leser sehr wichtigen Deux traités de physique mécanique, die eine von Prevost zusammengestellte Sammlung von vier Aufsätzen aus Lesages Nachlass enthält, erschienen erst 1818.

Prevosts Biographie zufolge hat sich Lesage bereits vor und während seines Studiums in Genf, wo er Physik bei Jean-Louis Calandrini (1703-1758) und Mathematik bei Gabriel Cramer (1704-1752) hörte ${ }^{20}$, mit dem Gravitationsproblem befasst und versucht, den freien Fall von Körpern auf Stösse schneller Atome zurückzuführen ${ }^{21}$. In Basel wurde er mit Daniel Bernoullis Atomphysik und Gastheorie bekannt ${ }^{22}$. Im Januar 1747 meldete er seinem Vater brieflich aus Paris, auf dem Wege zu einer mechanischen Erklärung des Abstandsquadratgesetzes und der Repulsion zu sein: Er habe eine neue Idee über den Mechanismus des Universums. Zwei Dinge hätten ihm Probleme bereitet: die Abstossung, die man zwischen den Partikeln einiger Elemente beobachte, und das Abstandsquadratgesetz. Beides habe er nun gelöst ${ }^{23}$.

Nach der Rückkehr in die Heimatstadt sollte ihm die neue Idee auch zu einem besseren Einkommen verhelfen. Er nahm 1748 und 1752 an zwei Preisaufgaben der Pariser Académie des Sciences zum Thema der Irregularitäten in den Bewegungen von Jupiter und Saturn teil. In seinen jeweils mit Essai sur l'origine des forces mortes überschriebenen Beiträgen ignorierte Lesage die Aufgabenstellung und stellte seine Gravitationstheorie vor. Die Preise gewann jedoch beide Male Leonhard Euler ${ }^{24}$. Erst die Teilnahme an der Preisaufgabe der Académie de Rouen Déterminer les Affinités qui se trouvent entre les Principaux Mixtes, ainsi que l'a commencé Mr Geoffroy; et trouver un Système Physico-méchanique de ces Affinités brachte 1758 den gewünschten Erfolg. Lesage gewann mit der Schrift Essai de Chymie méchanique den Preis für die Lösung des zweiten Teils der Aufgabe, Gewinner des ersten Teils war Jean-Philippe de Limbourg ${ }^{25}$. Dieser Essai enthält in Grundzügen die Gravitationstheorie Lesages, ist aber mehr noch den Phänomenen der mikrokosmischen Anziehung gewidmet, der Kohäsion, Kristallisation und Mischung, vor allem aber der chemischen Affinität.

20 Prevost $1805,16$.

21 Prevost $1805,30$.

22 Prevost 1805, 41.

23 «J'avois déjà depuis quatre ans une nouvelle idée sur le mécanisme de l'Univers: deux choses seulement m'embarrasaoient, l'explication de la répulsion qu'on observe dans les particules de certains élémens, et la loi du quarré des distances: or j'ai trouvé la permière de ces choses avant-hier, et la seconde il n'y a qu'un moment.» Prevost 1805, 51.

24 Evans 2002, 19.

25 Duncan 1996, 70, 90. 
Eine konzise und ausgereiftere Fassung der Gravitationstheorie bietet der 1784 veröffentlichte Lucrèce Neutonien. Einer Empfehlung Prevosts folgend $^{26}$, soll dieser Text als erstes untersucht werden, da er sich am besten dazu eignet, in die Prinzipien der Lesageschen Gravitations- und Materietheorie einzuführen.

\section{Der Newtonianische Lukrez}

Der stilistisch eigenwillige und reizvolle Aufsatz ist in Form einer Verteidigung der Epikureer gegen eine imaginäre Gruppe von Philosophen gestaltet und versetzt den Leser in eine zeitfreie Gedankenwelt, in der der mechanische Atomismus Epikurs, Demokrits und Lukrez' gegen die neuzeitliche Physik mathematischer Gesetze, für die Kepler, Galilei und Newton stehen, diskutiert wird. Der literarischen Form entspricht Lesages Leitmotiv, den antiken Atomismus als das geeignete Rüstzeug zur Beantwortung der Frage nach der Ursache bzw. dem Mechanismus der bislang nur als mathematische Gesetzmässigkeit beschriebenen Gravitationskraft zu präsentieren. Dazu bedurfte es Lesage zufolge jedoch einer gewichtigen Korrektur der alten Lehre. ${ }^{27}$

Am Beispiel der Bewegung des Mondes führte er aus, wie eine Abänderung der epikureischen Idee von einem nur nahezu parallelen Fall der Atome - der in der Vorstellung der antiken Atomisten als Auslöser von Atomkollisionen das schöpferische Moment aller Naturvorgänge bildete ${ }^{28}$ mit einem Schlag eine Reihe von Problemen löste. Die Epikureer hätten, so Lesage, die Atome mit Bewegungen ausstatten sollen, die senkrecht zur Oberfläche einer Kugel und damit auf ihr Zentrum gerichtet wären, ganz so, als ob es gleichzeitig auf der ganzen Erde hagelte ${ }^{29}$. Einen solchen radialen Teilchenstrom präsentierte er als Ursache der Gravitation. Auftreffende Atome würden von einem Himmelskörper abgefangen und übertrügen Impulse, die eine Bewegung des Himmelskörpers auslösten, wenn der Atomenstrom nicht aus jeder Richtung mit gleicher Intensität erfolgte. Dies geschähe, wenn ein anderer Himmelskörper in seiner Nähe stünde. Da die

26 Prevost 1805, 563.

27 Zum Motiv der prisca sapientia bei Newton siehe Boenke (2005).

28 Lukrez 1989, 11, 216-223: «Senken sich Körper geradenwegs dank eigener Schwerkraft / durch die Leere nach unten, so weichen sie, zeitlich wie örtlich / kaum zu bestimmen, etwas zur Seite, gerade so wenig, / daß man es noch als ein Ändern der Richtung darlegen könnte./ Wichen sie derart nicht ab, so müßten sie sämtlich, wie Tropfen / während des Platzregens, abwärts fallen ins bodenlos Leere. Keineswegs könnten die Urkörper dann auch sich treffen und stoßen, / niemals auch hätte sodann die Natur etwas geschaffen.»

29 Lesage 1805, 569. 
beiden Himmelskörper jeweils einen Teil des Stroms abfingen, träfe aus dem Raumausschnitt, in dem der jeweils andere Himmelskörper stünde, ein verminderter Strom auf. Die Himmelskörper drifteten folglich aufeinander zu.

Mit Hilfe dieses Modells entwarf Lesage eine Herleitung des Abstandsquadratgesetzes: Die Entfernung Erde-Mond beträgt 60 Erdradien. Demnach bewegt sich der Mond in einer Sphäre, deren Oberfläche 3600mal grösser ist als die Oberfläche der Erde. Die Anzahl der die Mondsphäre senkrecht treffenden, aufs Erdzentrum gerichteten Atome ist jedoch gleich gross der auf die Erdoberfläche auftreffenden. Damit ergibt sich für den Mond wegen der 3600fach geringeren Teilchendichte eine um den Faktor 1/3600 geminderte Schwere ${ }^{30}$.

Der die Gravitation hervorrufende radiale Korpuskelstrom stellte jedoch nur einen beliebigen Ausschnitt in einem im Prinzip völlig ungeordneten Kosmos geradlinig fliegender Gravitationskorpuskeln dar. Eine schöne und klare Beschreibung des Lesageschen Kosmos hat sein Schüler Pierre Prevost gegeben:

Stellen Sie sich den leeren Raum vor. Korpuskeln von extremer Kleinheit irren durch diesen Raum. Jede Korpuskel hat ihre bestimmte Richtung und bewegt sich geradlinig mit einer Geschwindigkeit, die bedeutend größer ist als die des Lichts. Die Richtungen dieser Korpuskeln sind so verschieden, sie sind so klein und ihre Geschwindigkeit ist derart groß, daß, obwohl der Raum nahezu leer ist, sie dennoch überall im Überfluß vorhanden sind. Das heißt, daß sie in einem sehr kurzen Augenblick einen beliebigen Punkt im Raum in alle Richtungen durchkreuzen, auch wenn dieser Punkt (den man sich als physikalischen Punkt denken muß) extrem klein wäre. Wenn sich zu irgendeinem Moment unsere Aufmerksamkeit auf irgendeinen dieser Punkte richtet, können wir feststellen, daß er das Zentrum einer unzähligen Menge von zusammenströmenden und auseinanderfliegenden Korpuskeln ist. Das bedeutet, daß in diesem Moment eine Vielzahl von Korpuskeln aus allen Punkten des Raums in diesem Punkt ankommen und daß genauso eine Vielzahl von Korpuskeln von dort in alle Richtungen ausgehen. ${ }^{31}$

Gegen den Einwand der imaginären Gegenpartei, dass sich unter der Annahme vollkommen regellos bewegender Gravitationsatome gar kein ausgezeichneter Punkt im Raum ergibt, der als Schwerezentrum fungieren könnte, verwiesen Lesages Epikureer darauf, dass derjenige Teil des Atomenstroms, der ein Auseinanderdriften bzw. einen Aufstieg fallender Körper verursachen würde, von der Dichte der Erde abgefangen würde ${ }^{32}$. Schwere war somit nichts, was einem Körper an sich zukäme, sondern er wurde erst schwer unter dem Hagel auftreffender Atome, die Lesage im Lucrèce Neutonien als atomes, corpuscules oder fluide gravifique bezeichnete ${ }^{33}$. Die von ihm

30 Lesage 1805, 571f. Mit der Angabe, dass die Teilchenzahl exakt gleich sei, macht Lesage einen Fehler, sie ist nur ungefähr gleich, da der Mond ja einen Teil der Atome abfängt.

31 Prevost 1818, Préface, XV-XVII.

32 Lesage 1805, 574.

33 Pierre Prevosts Übersetzer David Ludewig Bourguet übersetzt «gravifique» zutreffend, wenn auch etwas schwerfällig mit «schwermachend». Vgl. Prevost 1794, 29. 
ansonsten gern verwendeten Termini matière céleste und corpuscules ultramondains benutzte er hier nicht.

Der Umstand, dass die Bewegungen der Gravitationsatome als die Urbewegungen des Universums («mouvemens primitifs de l'univers») vollkommen ungeordnet und ohne ausgezeichnete Richtungen verliefen, bedurfte für Lesage aus philosophischen Gründen der Rechtfertigung. Er wies das System des antiken Atomismus mit seiner These des zufälligen Entstehens der Dinge als absurd und gottlos zurück ${ }^{34}$. Es ginge darum zu beweisen, dass und wie die Urbewegungen ein vollkommen geordnetes - und das bedeutete auch ein von einem göttlichen Wesen gewolltes - Weltsystem hervorbringen ${ }^{35}$. Zu diesem Zweck formulierte er zwei Propositionen, aus denen, wie er glaubte, sich die ganze Theorie der universellen Gravitation, das Newtonsche Gravitationsgesetz und - unter Berufung auf Newtons Prinzipien - die Keplerschen Gesetze $^{36}$ herleiten liessen:

1. Nur die Gravitationsatome, die zufällig auf das Zentrum irgendeines Körpers gerichtet waren, trugen zu dessen Schwere bei, nicht aber diejenigen, die den Körper nur seitlich passierten. Aus dieser Proposition folgte, wie am Beispiel des Mondes gezeigt, das Abstandsquadratgesetz.

2. Es waren nur diejenigen Gravitationsatome wirksam, die Impulse übertrugen und keine Antagonisten mehr hatten. Sie richteten sich nicht nur auf das Zentrum eines Himmelskörpers, sondern entfalteten ihre Wirksamkeit bei jedem Auftreffen auf dessen Materiepartikel. Die Schwere gegen das Zentrum eines Himmelskörpers war somit das Resultat unmerklicher Impulse gegen alle seine Teile, und daraus folgte, dass die Schwere proportional der Anzahl dieser Teile, und d.h. der Masse des Himmelskörpers, war $^{37}$.

Dennoch war das Problem der Massenabhängigkeit der Gravitation mit der zweiten Proposition keineswegs gelöst, denn die Hypothese der Atomimpulse implizierte eine Abhängigkeit des Gewichts von der Oberfläche und der Umgebung eines Körpers - ob er sich z.B. in einem geschlossenen Gebäude oder unter freiem Himmel befand - und führte damit zu einer Schlussfolgerung, die den Tatsachen widersprach. Um diesem Problem gerecht zu werden, sah sich Lesage zur Einführung eines weiteren materietheoretischen Prinzips gezwungen, das er unter Berufung auf eine eindrucksvolle Reihe antiker Autoren rechtfertigte: der weitgehenden Hohlheit und Durchdring-

34 Lukrez, De natura rerum, V 418-429.

35 Lesage 1805, 574, 594.

36 Lesage 1805, 583.

37 Lesage 1805, $577 \mathrm{f}$. 
barkeit der Körper. Die Materie sollte für die Gravitationsatome weitgehend durchlässig sein wie ein Diamant für Licht oder das Gold für den Magnetismus. Die Gravitationsatome vermochten durch ihre immense Geschwindigkeit, die er mit Lukrez höher als die Lichtgeschwindigkeit ansetzte, einen spürbaren Effekt auszuüben ${ }^{38}$.

Eine weitere Schwierigkeit des Gravitationsmechanismus bestand in möglichen Kollisionen der Gravitationskorpuskeln untereinander, da sie deren Verlangsamung und damit eine Schwächung der Gravitationskraft hervorrufen würden. Dies veranlasste Lesage zu einer näheren Bestimmung der Beschaffenheit seines Gravitationsfluidums. Er forderte eine geringe Teilchendichte, die wiederum nicht so gering werden durfte, dass eine zu kleine Zahl von Impulsen auf schwere Körper die Gravitationskraft diskontinuierlich erscheinen liesse. Diese Bedingung liess sich erfüllen, wenn man die Gravitationsatome extrem klein und darüber hinaus mit einer hohen Dichte ausgestattet dachte ${ }^{39}$.

In der posthum erschienenen Aufsatzsammlung Physique mécanique ergänzte Lesage diese Beschreibung der Gravitationsatome um die wichtige physikalische Eigenschaft ihrer vollkommenen Inelastizität, wodurch er die Stossvorgänge des Gravitationsmechanismus präziser erfasste ${ }^{40}$. Wohlweislich bezeichnete er sein fluide gravifique nicht als Äther, sondern reservierte diesen Begriff für die interkorpuskularen Wechselwirkungen der mikroskopischen Ebene, die Gegenstand der beiden letzten Abschnitte dieses Aufsatzes sind. Dennoch stellte er seine Theorie in den Kontext der Versuche anderer, im Lucrèce nicht namentlich genannter Physiker, die Vermittlung der Gravitation und die Ausbreitung des Lichts mit Hilfe von geradlinigen Bewegungen einer ätherischen Materie zu erklären. Die Abkehr von jeder Form von Wirbeltheorie war für ihn der entscheidende Punkt.

Je suis même très-convaincu que depuis qu'on connoît la loi que suivent les dégradations de la gravité universelle, sa conformité avec celle de la lumière aura fait penser à plusieurs physiciens qu'une matière éthérée, dont les mouvemens seroient rectilignes, pourroit bien être la cause de cette gravitation. ${ }^{41}$

38 Lukrez, De natura rerum, II 160-163. Lesage schätzte die Lichtgeschwindigkeit auf das 900000 fache der Schallgeschwindigkeit. Siehe Lesage 1805, 585, 588-590.

39 Lesage 1805, 591f.

40 Lesage 1818, 5. Zur Erläuterung: Beim vollkommen elastischen Stoss bleibt die Summe der kinetischen Energien erhalten. Ein näherungsweises Modell für den elastischen Stoss bietet der Zusammenprall zweier Billardkugeln. Beim vollkommen inelastischen Stoss dagegen bleiben beide Körper nach dem Stoss zusammen und bewegen sich mit der gemeinsamen Geschwindigkeit $\mathrm{v}=\left(\mathrm{m}_{1} \mathrm{v}_{1}+\mathrm{m}_{2} \mathrm{v}_{2}\right) /\left(\mathrm{m}_{1}+\mathrm{m}_{2}\right)$ weiter. Dabei tritt der Carnot-Energieverlust ein, d.h., ein Teil der kinetischen Energie wird in Wärme umgewandelt.

41 Lesage 1805, 597. 
Bei diesen «Physikern» handelt es sich um Nicolas Fatio de Duillier (16641753), Gabriel Cramer ${ }^{42}$, Frantz Albert Redeker, einen deutschen Mediziner, der 1736 ein Werk De causa gravitatis meditatio publiziert hatte, und Johann Hudde (ca. 1633-1704), Mathematiker und Bürgermeister von Amsterdam. Lesage hat diese Namen in seiner unveröffentlicht gebliebenen Histoire de la pesanteur genannt ${ }^{43}$.

Im Begriff der corpuscules gravifiques als der Himmelsmaterie mit ihren Merkmalen der - wenn auch nur diskreten - Raumerfüllung, der permanenten Bewegung und der unmittelbaren Beziehung zur Gottheit als Urkraft hinter allen Naturvorgängen blieben so die Charakteristika des antiken Ätherbegriffs erhalten ${ }^{44}$. Wenn Lesage jedoch den Terminus Äther für die Kräfte im Mikrokosmos reservierte, hielt er dabei seinem antiken Vorbild Lukrez die Treue, da auch bei ihm der Äther lediglich eine nachgeordnete Form subtiler Materie darstellte, nicht aber eine schöpferische Urkraft ${ }^{45}$.

\section{Kraftbegriff und Wirbeltheorie: die Korrespondenz mit Euler}

Mit seiner gleichzeitigen Zurückweisung des newtonianischen Kraftbegriffs ${ }^{46}$ und der Wirbeltheorie nahm Lesage eine mittlere Position zwischen den Vertretern eines dynamischen Korpuskularismus und den Anhängern der mechanistischen Physik ein. Die erste Gruppe, von Leonhard Euler (1707-1783) als Attraktionisten bezeichnet, fand ihre meisten Mitglieder in England, doch auch auf dem Kontinent bekannten sich einflussreiche Naturwissenschaftler und Philosophen wie Georges Louis Leclerc Comte de Buffon (1707-1788), Voltaire (1694-1778) oder Denis Diderot (1713-1784) zu einer Variante des Newtonianismus, die die Gravitation als materieinhärente Kraft auffasste und die Möglichkeit der Fernwirkung zuliess. Dagegen hatten führende Physiker und Mathematiker auf dem Kontinent unter Anerkennung der von Newton formulierten mathematischen Gesetzmässigkeiten an den Grundzügen der cartesischen Physik und Kosmologie und damit auch der Wirbeltheorie festgehalten ${ }^{47}$. Einer der wichtigsten

42 Zehe 1980, 279f. und passim. Nach Zehes Analyse ist Cramers Theorie vollständig von Fatios abhängig.

43 Stosz 1884, 17, 47-49; Prevost 1818, XXIX-XXXIII.

44 Cicero, De natura deorum II, 28,32,42, 64,91. Lukrez, De rerum natura II, 290-301;1050-1053.

45 Bei Lukrez kommt dem Äther in erster Linie die Bedeutung der feurigen Himmelsmaterie zu. Von der Idee des Feueräthers als schöpferischer Urkraft distanzierte er sich ausdrücklich. Lukrez, De rerum natura I, 700-710.

46 Rosenberger 1887-1890, 19.

47 Boenke 2004. 
Vertreter dieser Denktradition war Lesages älterer Zeitgenosse Leonhard Euler, dessen Korrespondenz mit Lesage seit kurzem vollständig ediert vorliegt ${ }^{48}$. Die Korrespondenz umfasst insgesamt neun Briefe, die die beiden Wissenschaftler in einem Zeitraum von 15 Jahren zwischen 1761 und 1776 austauschten. Sie wurde von Lesage mit der Übersendung eines Exemplars des Essai de Chymie méchanique eröffnet und erst auf seine nachdrückliche Aufforderung hin von Euler auf dem Niveau eines echten wissenschaftlichen Austauschs weitergeführt.

Im Essai hatte Lesage Newtons Begriff der Kraft, den er ganz im Sinne des dynamischen Korpuskularismus verstand, in einer polemischen Wendung als hohle Idee und metaphysisches Etwas verworfen.

Je suis surpris: Que Mr. Newton; qui sentoit si bien toute la solidité de l'Analogie ...; se soit si fort pressé ici, d'en faire application à l'idée creuse de Force en gènèral (Etre Métaphysique, pour ne rien dire pis); plutôt que de s'attacher immèdiatement, au Genre prochain de cette Analogie, savoir l'Impulsion. ${ }^{49}$

Lesage mass dem Analogieschluss eine hohe Bedeutung bei. Vorausgesetzt war dabei der Satz, dass ähnliche Wirkungen aus ähnlichen Ursachen hervorgehen, den er in den Rang eines Axioms hob. Keine Beweismethode schien ihm besser geeignet, Naturvorgänge jenseits des sinnlich Fassbaren zu begreifen. «Nous n'avons aucune preuve de ce qui se passe hors de nous, plus forte que l'est l'Analogie.» ${ }^{50}$ Ein strenger Analogieschluss beweise die Reduzierbarkeit aller Zustandsänderungen auf Stossvorgänge. Unter den Begriff der Zustandsänderung waren dabei von vornherein nur solche des Bewegungszustands gefasst: Übergänge von Ruhe in Bewegung und umgekehrt, Beschleunigung und Verzögerung, abrupte und allmähliche Richtungsänderungen. Einfache Beobachtung, so Lesage, zeige die unmittelbare Verursachung von Zustandsänderungen durch Stösse anderer Körper, daher gelte dasselbe für unsichtbare Ursachen wie die Gravitation. «Donc, l'Approche mutuëlle des Corps visibles, est duë à l'Impulsion immèdiate de quelque Matière invisible. ${ }^{51}$ Dieses Argument lieferte die Rechtfertigung seines mechanistischen Ansatzes und brachte ihn dem Eulerschen Lager der Impulsionairs nahe ${ }^{52}$.

So bleibt als nächstes zu klären, worauf seine Kritik anspielte, wenn er die Impulsionen als das von Newton übergangene Genus proximum bezeichnete. Bemerkenswerterweise zog Lesage in diesem Zusammenhang unter Beru-

48 Kleinert (im Druck).

49 Lesage o.J., 15.

50 Lesage o.J., 14 (kursiv im Original).

51 Lesage o.J., 14.

52 Euler 1986, 54. Brief. 
fung auf die zeitgenössische Biologie einen traditionsreichen Analogieschluss heran ${ }^{53}$ : So wie die willkürlichen Bewegungen der Menschen in einer Kausalkette von den Muskelbewegungen über Nerven und Gehirnaktivität zuletzt auf die Seele als immaterielle Wesenheit zurückgehen, nehmen auch die sichtbaren Bewegungen in der Natur über die Vermittlung einer unsichtbaren Materie ihren Ursprung letztlich aus einem immateriellen Wesen. Damit waren die Gravitationskorpuskeln in eine der klassischen Funktionen des Äthers eingesetzt, nämlich die der Vermittlung zwischen dem Immateriellen als aktivem und dem Materiellen als passivem Prinzip.

Anders als die cartesianischen Physiker beharrte Lesage auf der Geradlinigkeit der Urbewegungen. Sie ergab sich unter Voraussetzung des Trägheitsgesetzes als logische Konsequenz aus der postulierten Dünnheit der subtilen Materie.

Tout mouvement Curviligne, étant forcé; \& tendant perpètuellement à devenir Rectiligne, dès l'instant que ce qui le gêne sera enlevé: Un pareil mouvement, ne peut pas subsister deux instans, dans un Fluide dont les parties ne se gênent point, \& peuvent s'èchaper ailleurs. Donc, le mouvement de nôtre Fluide, est actuellement Rectiligne. ${ }^{54}$

Zum Vergleich verwies Lesage weiterhin auf die Geradlinigkeit der Lichtausbreitung. Gravitation wie Licht folgten gleichermassen dem Abstandsquadratgesetz und sollten sich daher auch in der Weise ihrer Ausbreitung ähneln ${ }^{55}$.

Euler begrüsste zwar grundsätzlich Lesages Bekenntnis zur mechanistischen Physik, doch schwang gleichzeitig Skepsis mit gegenüber der Behauptung des jüngeren, weniger profilierten Kollegen, das Gravitationsproblem gelöst zu haben. «Quoique je fusse fort éloigné de m'imaginer, que je puisse decouvrir la veritable cause mecanique, je n'ai jamais douté de son existence.» ${ }^{56}$

Lesages Beweis der Geradlinigkeit der Urbewegungen aus der Analogie der Lichtausbreitung fand Eulers scharfe Kritik. Der Newtonschen Emanationstheorie hatte er eine Theorie entgegengesetzt, die das Licht als eine Bewegung oder Erschütterung in den kleinsten Teilchen des Äthers interpretierte ${ }^{57}$. Im Hinblick auf Lesages Gravitationstheorie ist an dieser Stelle vor allem folgendes Argument Eulers gegen Newtons Lichtteilchen von Belang: Bei allen leuchtenden Himmelskörpern, die man auf einmal sieht, müsste ein Durchkreuzen der Lichtstrahlen stattfinden. Der Umstand, dass

53 Aristoteles, De mundo 399b; Platon, Philebos 30a-e.

54 Lesage o.J., 17.

55 Lesage o.J., 18.

56 Kleinert (im Druck), Euler à Lesage, Berlin, 16 avril 1763.

57 Euler 1986, 20. Brief. 
sie trotzdem klar und deutlich erkennbar blieben, zeigte, dass mehrere Lichtstrahlen durch denselben Punkt gehen konnten, ohne einander zu stören. Dies widerlegte nach Eulers Ansicht die Emanationstheorie ${ }^{58}$, und es ist zweifellos dieses Argument, auf das er anspielte, wenn er Bedenken äusserte, ob Lesages System der unendlich vielen, sich kreuzenden Korpuskularströme auch nur einen Moment bestehen könne.

Sein zweiter Einwand galt Lesages Reduktion aller mechanischen Wechselwirkungen auf Stossvorgänge. Er sah darin den Fall der gehinderten Bewegung nicht berücksichtigt. Genau um eine solche handelte es sich jedoch bei den gekrümmten Bewegungen, wie sie z.B. eine Schleuder verursachte. Hier liess sich schliesslich das Auftreten von Zentrifugalkräften beobachten. Euler konzedierte zwar Unzulänglichkeiten der Wirbeltheorie, hielt aber an ihrem Prinzip fest und setzte Lesages Gravitationsimpulsionen die Vorstellung einer von einem plenistischen Ätherfluidum durch kontinuierlichen Druck verursachten Bewegung entgegen.

Il est plutot certain que la matiere subtile, qui remplit tous les espaces parmi les corps celestes pourroit se trouver dans une telle agitation, que les pressions seroient partout tellement inegales entr'elles, que les corps, qui y sont plonges en seroient poussés de la meme maniere que dans l'hypothese de l'attraction. ${ }^{59}$

Trotz ihres übereinstimmenden Anspruchs, eine mechanistische Physik zu betreiben, zeigt die Euler-Lesage-Korrespondenz in anschaulicher Weise die grundsätzlichen Differenzen zwischen Lesages Mechanik und der der späten Cartesianer: geradlinige Urbewegungen in einem weitgehend leeren Raum versus Wirbelbewegungen eines plenistischen Ätherfluidums, Reduktion aller Zustandsänderungen auf Impulsionen versus nichtgeradlinige Bewegungen durch kontinuierlichen Druck des Äthers und nicht zuletzt das Bekenntnis zur Korpuskulartheorie des Lichts gegenüber der Undulationstheorie.

\section{Der Aufbau der schweren Materie}

Dem im Lucrèce Neutonien eingeführten Postulat einer weitgehend leeren und durchlässigen Materie, das der ältere Essai de Chymie méchanique mit

58 Euler 1986, 17. Brief.

59 Kleinert (im Druck), Euler à Lesage, Berlin, 16 avril 1763. Im Brief vom 8. September 1765 führt Euler diesen Gedanken noch einmal aus: «... il se trouve dans le monde outre l'ether encore un autre fluide incomparablement plus subtile et plus elastique, où il faut sans doute chercher la cause de la gravitation universelle; or cela non pas par des chocs réels, comme Vous supposés à Vos corpuscules ultramondains, mais par de simples pressions telles que nous connoissons dans tous les fluides.» 
seiner unschärferen Rede von Poren noch nicht enthält, hat Lesage in einem Appendix zum Lucrèce und in einem Text der Physique mécanique aus dem Jahre 1767 breiteren Raum gewidmet. Dennoch kam er nicht über Ansätze hinaus. Eine kohärente Theorie über Aufbau und Struktur der schweren Materie hat er nicht vorgelegt. So kam er bei einer Reihe von Fragen zu keinem klaren Urteil. Im Lucrèce dachte er die unteilbaren Partikel der schweren Materie in Gestalt von Käfigen, z.B. hohlen Kuben oder Oktaedern. Die Durchmesser der Stäbe dieser Käfige sollten so klein sein, dass der Erdglobus insgesamt weniger als den 10000 sten Teil der ihn durchströmenden Gravitationskorpuskeln auffangen sollte. Die Durchmesser der Balken unterschieden sich nur minimal voneinander ${ }^{60}$.

Definitive Aussagen über die Formen der Käfige und Stäbe wollte er nicht treffen $^{61}$. Er versuchte lediglich, mit Hilfe mathematischer Spekulationen die ungeheure Dünnheit der Materie grössenordnungsmässig zu erfassen. So errechnete er z.B. unter der Voraussetzung einer maximalen Abfangrate der Erde von $1 / 20000$ der durchströmenden Gravitationskorpuskeln und einer gedachten Unterteilung der Erde in 500 Millionen parallele Platten im jeweiligen Abstand von einem Zoll für die Durchmesser der Polyederstangen der schweren Materie einen Wert von einem zehnbillionstel Zoll ${ }^{62}$. Dieser Wert erwies sich aber im Vergleich zu anderen Kalkulationen mit anderen Plattenzahlen und Abständen als in so hohem Mass abhängig vom jeweils zugrunde gelegten Modell, dass er vollkommen willkürlich erscheinen musste. Daher kam Lesage zu keiner klaren Schlussfolgerung. Die Gestalt der schweren Korpuskeln konnte faktisch nur als reine Rechengrösse behandelt werden, die sich je nach Bedarf festlegen liess. So mochte es unter Umständen durchaus sinnvoll erscheinen, sie kugelförmig anzunehmen:

Les élémens des graves doivent donc être conçus comme des cages, dont les barreaux (supposés cylindriques) sont d'un diamètre très petit, relativement à leur longueur, et laissent entr'eux des intervalles immenses, par rapport à ce même diamètre.

Cependant lorsqu'il s'agit d'apprécier les effets de la percussion des corpuscules contre ces élémens, il sera plus simple de réduire les particules frappées à la forme sphérique ...63

Damit bleibt letztlich die weitgehende Leerheit der schweren Materie als das wesentliche und durchgängige Merkmal seiner hochspekulativen Materietheorie festzuhalten. Mit dieser Position steht Lesage im 18. Jahrhundert nicht allein. Einer Untersuchung Thackrays zufolge gewann diese Idee, die Thackray im Rückgriff auf Joseph Priestleys zugespitzte Bemerkung - «all the solid matter in the solar system might be contained within a nut-shell»-

60 Lesage 1805, 599f. Lesage 1818a, 11. Zur Datierung dieses Textes siehe S. 52, Fussnote.

61 Lesage 1818, 46.

62 Lesage 1818, 53.

63 Lesage 1818, 59. 
als Nut-shell-Theorie bezeichnet, unter den frühen Newtonianern und bei Newton selbst in der fortdauernden Kontroverse mit Leibniz zunehmend an Bedeutung $^{64}$. Im Briefwechsel zwischen Clarke und Leibniz wurde sie im Hinblick auf ihre theologische Relevanz diskutiert. So referierte Leibniz die Nut-shell-Theorie im zweiten Brief mit Selbstverständlichkeit als den Standpunkt Newtons:

Man behauptet weiter, daß nach den mathematischen Prinzipien, d.h. nach der Philosophie Newtons - denn die mathematischen Prinzipien machen darüber nichts aus - die Materie der unbedeutendste Teil des Universums ist. Newton nämlich nimmt außer der Materie einen leeren Raum an, nach ihm nimmt also die Materie nur einen sehr kleinen Teil des Raumes ein. ${ }^{65}$

Schon 1702 hatte der Newton-Schüler John Keill behauptet, es sei prinzipiell möglich, ein Korn solider Materie so zu zerstreuen, dass sie jeden noch so grossen, begrenzten Raum erfüllte. Später konkretisierte er seine Vorstellung mit der Angabe, dass die Quantität der Materie im Gold zwar 20 000mal grösser sei als die in der Luft, das Verhältnis der darin enthaltenen leeren Räume dagegen eher in einem Verhältnis von 999999 zu 1000000 stünde. David Gregory interpretierte das Phänomen der verschieden hohen Dichten von Stoffen, etwa von Wasser und Gold, als Hinweis auf eine beachtliche Porosität der Materie. Auch die Royal Society war mit dem Problem befasst. Einen von John Theophilus Desaguliers 1714 vorgeführten Versuch, der die nahezu vollständige Lichtdurchlässigkeit von Glaslinsen zum Gegenstand hatte, wertete man als Beweis der Dünnheit der Materie ${ }^{66}$.

Schliesslich hatte Newton selbst im dritten Teil des zweiten Buches der Optik von 1717 über die Struktur der porösen Materie räsoniert und eine mehrstufige Ordnungsstruktur von Poren und Hohlräumen vorgeschlagen, die ihre extreme Dünnheit plausibel machen sollte ${ }^{67}$. Diese Überlegungen waren Lesage bekannt und wurden von ihm im Essai referiert.

Or, s'il y a de la sorte, plusieurs Ordres successifs de Cloisons; \& que dans chaque Ordre, la moitié du volume soit occupée par des Pores aussi grands que les Cloisons de l'ordre suivant: La quantité réelle de Matiére, ne sera que la moitié, le quart, la $1 / 8,1 \mathrm{la} 1 / 16$, \&c. de tout le volume. Dix semblables ordres, par exemple; feront que la Masse ne sera que la $1 / 1024$ partie du volume. C'est la façon dont Mr. Newton conçoit que les Corps sont composés. ${ }^{68}$

Doch über die grundsätzliche Feststellung der weitgehenden Leerheit der Materie durch die grösste Autorität seines Fachs hinaus liess sich daraus für Lesage im Detail nicht viel gewinnen. Ganz anders liegt der Fall bei seinem

64 Thackray 1970,54. Die folgende Darstellung beruht auf diesem Buch und auf Thackray 1968. 65 Leibniz 1966, $124 \mathrm{f}$.

66 Thackray 1968, 38.

67 Thackray 1970, 53-67.

68 Lesage o.J., 8. 
älteren Basler Landsmann Fatio de Duillier, der als Mitglied der Royal Society die intellektuelle Atmosphäre des Newtonianismus genossen hatte. Die Gravitations- und Materietheorien Lesages und Fatios weisen grundlegende Übereinstimmungen auf, so dass der Vorwurf des Plagiats nahelag und auch erhoben wurde. Gegen ihn hat sich Lesage in zahlreichen Fussnoten und in Form von schriftlichen Erklärungen seiner Schüler wiederholt gewehrt $^{69}$. An dieser Stelle soll unter Zugrundelegung der Darstellung Horst Zehes, und dem folgenden Abschnitt etwas vorgreifend, dieser Vorwurf zunächst anhand des Problems der Materiestruktur geprüft werden ${ }^{70}$.

Die Annahme der Porosität und Leere der Materie war für Fatio - wie für Lesage - ein Erfordernis der Gravitationstheorie. Es galt den Widerspruch aufzulösen, dass einerseits die Gravitationskraft von der Masse der Körper abhing, andererseits die von beiden verfochtene Theorie der Impulse von Gravitationskorpuskeln eine Abhängigkeit von der Körperoberfläche implizierte. Die Struktur der Materie musste also so beschaffen sein, dass Oberfläche und Masse richtungsunabhängig zueinander proportional waren, damit die aus allen Richtungen auftreffenden Gravitationskorpuskeln aus jedem Blickwinkel dieselbe Zahl von Impulsen übertrugen ${ }^{71}$.

Fatio de Duillier entwarf und verwarf verschiedene Modelle, die diesen Bedingungen genügen sollten. Er entschied sich schliesslich für eine Struktur, die die Kriterien der Dünnheit und Kugelsymmetrie am besten zu erfüllen schien:

Man stelle sich wie oben ein Gitter oder Netz vor, gleichgültig von welcher Gestalt und welcher Seitenzahl - die größte scheint jedoch die beste. In dessen Mitte denke man sich eine durch starre Stäbe aufgehängte oder befestigte Kugel. Der Durchmesser der Kugel ist unendlich kleiner als der des gesamten Netzes, und der Durchmesser der Fäden, die das Netz und die Stäbe zusammensetzen wiederum unendlich kleiner als derjenige der Kugel, und die Oberfläche - und umsomehr die Masse - des Netzes unendlich kleiner als die der Kugel. Ein Haufen solcher Atome bildet eine im Verhältnis zu seiner Dichte attraktive Masse.72

Auch Lesage unternahm Versuche, eine Struktur zu ersinnen, die die Richtungsunabhängigkeit der Masse eines Körpers einleuchtend erklärte. Die Teilchen eines Körpers, schrieb er, könnten verschiedene Formen besitzen, und selbst wenn sie unter sich gleich wären, könnten sie relativ zum Strom der corpuscules ultramondains unterschiedliche Lagen einnehmen. Eine unbestimmte Form von Polyederstruktur war von ihm hierbei vorausgesetzt. Die Aufgabe bestand also darin, zu bestimmen, unter welchen Bedingungen sich den einströmenden Gravitationskorpuskeln aus allen Richtungen die-

69 Prevost 1805, 164f., 339f. Prevost 1818, XXXIII.

70 Zehe 1980.

71 Zehe 1980, 209.

72 Zehe 1980, 37; zitiert nach Zehe 1980, 212. 
selbe Zahl von Eckpunkten und Flächen darbietet ${ }^{73}$. Sein Lösungsvorschlag bestand, anders als bei Fatio, in einem lediglich mathematischen Modell aus Punkten, Geraden und Sphären. Doch erinnert dieses Modell so sehr an das von Fatio, dass eine Abhängigkeit Lesages in diesem Punkt angenommen werden muss.

... un très-grand nombre de droites égales, qui partent d'un même point, font en tous sens des angles égaux avec leurs voisines, et qu'il n'importe pas qu'on change le plan où chacune d'elles est placée, pourvu qu'on ne change pas leur longueur ni ce sommet commun, ni l'angle que cette droite fait avec un certain axe commun ...

Les extrémités de toutes ces droites sont dans la surface d'une même sphère, et elles y sont parsemées uniformement; de sorte que, dans différentes portions de cette surface, les nombres de ces points sont proportionnels à ces portions. ${ }^{74}$

Es wäre allerdings nicht angebracht, Lesage den Versuch des Plagiats zu unterstellen, da er seine Kenntnis der Fatioschen Theorie der schweren Materie und seine Übereinstimmung damit zugestand ${ }^{75}$.

\section{Zum Vorwurf des Plagiats: die Gravitationstheorie Fatio de Duilliers}

Die wissenschaftshistorischen Untersuchungen von Zehe und Gagnebin haben gezeigt, dass Lesage spätestens seit 1749 - d.h. zwar einige Jahre vor der Niederschrift des Essai de Chymie méchanique, aber auch ein Jahr nach der erstmaligen Präsentation seiner Lösung des Gravitationsproblems für die Pariser Akademie im Essai sur l'origine des forces mortes ${ }^{76}$ - über seinen Lehrer Gabriel Cramer zumindest indirekte Kenntnis der Arbeiten Fatio de Duilliers besass. Cramer hatte ferner 1731 in Genf unter dem Titel Theses Physico-Mathematicae de Gravitate eine Abhandlung verfasst, die Fatio de Duillier als Plagiat aufgefasst haben muss. So schrieb er über Cramer, dass er «meine Theorie in öffentlichen Thesen verkürzt dargestellt und unter seinem Namen verbreitet hat, ohne sie recht zu verstehen ${ }^{77}$. Seit 1761 bemühte sich Lesage aktiv und mit einigem Erfolg um den Besitz einiger Originalmanuskripte. Es gelang ihm, einige Fragmente Fatios über die Schweretheorie aufzutreiben. In seinem Nachlass fanden sich ferner Auszüge aus dessen Korrespondenz, Briefe von Huygens, Leibniz und Jakob Bernoulli

73 Lesage 1818, 48.

74 Lesage 1818, 49 f.

75 Lesage 1818, 45: «Je penche à présent beaucoup à croire, avec M. Fatio, que les élémens sont des espèces de cages, ou des polyèdres réduits à leur côtés ou arrêtes; et que ces côtés sont des fils dont l'épaisseur est prodigieusement moindre que la longueur, par exemple, $1: 10^{20}$.»

76 Prevost 1805, 67.

77 Zitiert nach Zehe 1980, 279f. 
an Fatio sowie Entwürfe seiner Antwortschreiben, in denen Fragen der Gravitationstheorie diskutiert wurden ${ }^{78}$.

Neben Versuchen einer historischen Rekonstruktion der Lesage zur Verfügung stehenden Informationsquellen bietet ein direkter Vergleich beider Theorien eine weitere Möglichkeit, die Berechtigung des Plagiatsvorwurfs zu prüfen. Ein solcher Vergleich wurde bisher noch nicht vorgenommen. $\mathrm{Zu}$ seinem Zweck kann neben der schon erwähnten Arbeit Horst Zehes auf zwei Editionen verwiesen werden. Die umfangreichste Fassung der Fatioschen Theorie bietet dabei die 1929 von Karl Bopp vorgenommene Herausgabe eines Manuskripts der Universitätsbibliothek Basel aus dem Nachlass der Familie Bernoulli. Daneben hat Bernard Gagnebin 1949 aus einigen Fragmenten der Genfer Bibliothek eine kürzere Fassung De la cause de la pesanteur rekonstruiert, die wesentliche Teile der Bopp-Edition, nämlich Texte zum Problem der Stosskraft der schwermachenden Materie («Problem II»), zum Unendlichen («Problem III») und zum Widerstand in der schwermachenden Materie («Problem IV»), nicht enthält ${ }^{79}$.

Fatio de Duilliers Gravitationstheorie unterschied, wie diejenige Lesages, zwei Materiearten: die der Gravitationskraft unterliegende grobe Materie und die Matiere, qui cause la Pesanteur, von Fatio unmittelbar mit dem Äther identifiziert, der die Anziehung zwischen der groben Materie hervorrief, ihr aber nicht unterlag ${ }^{80}$. Der Weltraum war erfüllt von dieser äusserst dünnen Äthermaterie, deren Partikel sich mit ausserordentlicher Geschwindigkeit in alle Richtungen bewegten. Dabei war der Weltraum als nahezu leer anzusehen $^{81}$. In ihm bewegten sich die Gravitationsteilchen in praktisch nicht wahrnehmbaren Zeiträumen aus den Fixsternregionen zur Erde ${ }^{82}$. Das Weltall war in einem absoluten Sinne als unendlich gross anzusehen.

L'Espace est Infini proprement, et à la rigueur. Cest à dire qu'il est non seulement infiniment plus grand, que le Globe de la Terre, par example: mais encore qu'il n'a aucunes Bornes, d'aucun côté que ce soit; et qu'il n'y a aucune Etendue, qui lui puisse être ajoutée, et laquelle il ne comprenne pas déja. ${ }^{83}$

Es findet sich in diesen Formulierungen Fatios keinerlei Anklang an eine Vorstellung, die Lesages Begriff ultramondain nahekäme.

Anders als dessen corpuscules gravifiques bzw. ultramondains war Fatio de Duilliers Gravitationsäther heterogen konzipiert. Seine Partikel unter-

78 Zehe 1980, 59-63; Gagnebin 1949,118-122. Diese Edition enthält die beiden Huygensschen Briefe.

79 Bopp 1929.

80 Gagnebin 1949, 130.

81 Gagnebin 1949, 127.

82 Gagnebin 1949, 128.

83 Gagnebin 1949, 134. 
schieden sich in Gestalt, Grösse, Geschwindigkeit, Rotationsbewegung und Federkraft. Vor allem dieses letzte Kriterium hatte, wie im folgenden gezeigt werden soll, bedeutende Konsequenzen für die Physik des Gravitationsmechanismus, der sich von demjenigen Lesages markant unterschied.Zudem stand Fatio de Duilliers Materietheorie dem Cartesianismus noch wesentlich näher, wenn er eine Vielzahl von Ordnungen von Materien postulierte, die sich durch ihren Grad an Feinheit und Beweglichkeit unterschieden. In diesem Zusammenhang war es vor allem die Federkraft (ressort) bzw. Elastizität der Partikel, die ihn interessierte. Die Teilchen des Äthers als die Teilchen der letzten Materieordnung sollten zwar, wie bei Lesage, vollkommen hart sein, doch war in Fatios System diese irreduzible Qualität mit einer nahezu vollkommenen Elastizität verbunden, während Lesages Gravitationskorpuskeln absolut inelastisch sein sollten.

... les dernieres Parties de la Matiere, lesquelles necessairement sont infiniment dures, aient un Ressort à peu près parfait, ou qui aproche infiniment d'étre parfait, et dont la Cause soit Metaphysique, et n'ait son Fondement, que dans la Volonté du Createur. ${ }^{84}$

Kern der Fatioschen Gravitationstheorie bildete das sogenannte Problem I, das den Mechanismus der Schwerkraft behandelt. Fatios Gedankenmodell postulierte einen vollkommen soliden, für die Äthermaterie undurchlässigen Körper $\mathrm{C}$ im unendlichen Raum. C war umgeben von den sehr dünn und gleichmässig verteilten Ätherteilchen, die sich geradlinig und unbehindert mit unterschiedlichen Geschwindigkeiten gleichermassen in alle Richtungen bewegten. Somit trafen sie auch gleichmässig aus allen Richtungen auf den Körper C auf, der folglich unbewegt blieb ${ }^{85}$. Auf der Oberfläche von $\mathrm{C}$ wählte er einen infinitesimalen Flächenausschnitt ZZ, der den Mittelpunkt einer Kugel bildete. Von ZZ als (stumpfer) Spitze ausgehend, wurde die Kugel in eine begrenzte Zahl von Pyramiden geteilt, deren eine, paradigmatisch ausgewählt, Fatio mit PZZQ bezeichnete. P und Q waren Punkte der Kugel. Die Bezeichnung von Flächen mittels zweier Punkte, d.h. als Strecken, erklärt sich aus der Zweidimensionalität seiner geometrischen Darstellung ${ }^{86}$. Die Seiten der Pyramide ins Unendliche verlängert gedacht, folgerte Fatio de Duillier, dass es in einem derartigen Raumausschnitt eine unendliche Zahl von Ätherkorpuskeln gäbe, die sich kontinuierlich der Länge der Pyramide entlangbewegten und schliesslich in $\mathrm{ZZ}$ aufträfen ${ }^{87}$. Die Gesamtzahl der aus allen Richtungen in ZZ auftreffenden Ätherkorpuskeln konstituierte einen Teil-

84 Gagnebin 1949, 130.

85 Gagnebin 1949,138; vgl. Bopp 1929, 32f., wo nicht von unterschiedlichen Geschwindigkeiten die Rede ist.

86 Vgl. Bopp 1929, Tafel I, Fig. I sowie Zehe 1980,143.

87 Gagnebin 1949, 139. Vergleiche Bopp 1929, 33. 
chenstrom, dessen Kraft reziprok zum Abstandsquadrat abnahm ${ }^{88}$. Im Unterschied zu Lesage nahm er für den Gravitationsmechanismus einen nahezu vollkommen elastischen Stoss an. Die Ätherkorpuskeln sollten im Augenblick des Stosses einen kleinen Teil ihrer Geschwindigkeit verlieren, so dass die reflektierten Teilchen sich etwas langsamer bewegten. Die Differenz dieser Geschwindigkeiten bildete die Ursache der Schwerkraft: «... le Courant qui vient contre ZZ, est généralement plus fort que celui qui s'en éloigne; Et que, par l'Excès de sa Force, il produit une Pesanteur tendant vers ZZ.» ${ }^{89}$

Lesage selbst hat die Elastizität der Fatioschen Gravitationskorpuskeln neben seinem eigenen Begriff der regions ultramondaines als den wesentlichen Unterschied beider Theorien herausgestellt ${ }^{90}$. Dagegen haben Zehe und Evans betont, dass bei beiden Autoren gerade die Inelastizität des Gravitationsstosses das schwereerzeugende Moment darstelle. Dabei unterschiebt Zehe pikanterweise Fatio de Duillier eine Formulierung des Gravitationsmechanismus, die nun tatsächlich von Lesage stammt, nämlich die sowohl im Lucrèce Neutonien wie im Essai de Chymie méchanique vorgetragene Theorie der Abschirmung von Gravitationsströmen durch zwei Himmelskörper als Ursache ihrer gegenseitigen Anziehung ${ }^{91}$. Gerade hierin liegt aber die eigentliche Differenz ihrer Systeme. Für Fatio de Duillier kam Schwere einem Körper allein zu, sie wurde durch eine geringe Inelastizität der Gravitationsstösse verursacht, die einen kleinen Unterschied in den Geschwindigkeiten der auftreffenden und der reflektierten Ätherteilchen zur Folge hatte. Für Lesage dagegen war die Schwere ein bloss relatives Phänomen, das nur zwischen mindestens zwei Körpern auftreten konnte, wobei der Abschirmeffekt nicht auf einer Reflexion, sondern auf einer Absorption von Gravitationskorpuskeln beruhte.

\section{Die Kosmologie der corpuscules ultramondains}

Lesage hat für seine Gravitationsatome den eigentümlichen Terminus corpuscules ultramondains geprägt. Dieser geheimnisvolle Ausdruck wirft die Frage nach der Bedeutung des Wortes ultramondain, nach dem Ursprung dieser Partikeln und eventuellen Konsequenzen für die Kosmologie auf. Die Wissenschaftshistoriographie hat diesem Aspekt der Schriften Lesages

88 Gagnebin 1949, $140 f$.

89 Gagnebin 1949, 142.

90 Prevost 1805, 67f.

91 Zehe 1980, 54. 
bisher praktisch keine Beachtung geschenk ${ }^{92}$, was seinen Grund darin finden mag, dass Lesage selbst sich relativ selten und nur knapp zu diesem Thema geäussert hat. Im Essai de Chymie méchanique erklärte er z.B. ultramondain als «jenseits der sichtbaren Welt» - «au dela du Monde visible»-, und er verband damit eine Betrachtung über Schöpfung, Grösse und Alter des Universums. Die corpuscules ultramondains wurden, so Lesage, im Moment der Schöpfung vor einigen Tausend Jahren ausgeschleudert und fliegen seither in geradliniger Bewegung durch das Weltall. Da sie sich mit extrem hoher Geschwindigkeit bewegen, haben diejenigen Korpuskeln, die heute auf die Erde auftreffen, ungeheuer weite Strecken zurückgelegt. Folglich wurden sie in kosmischen Regionen ausgeschleudert, die von der Erde ausserordentlich weit entfernt waren. In diesem Zusammenhang flocht er eine teleologische Betrachtung ein, die gleichzeitig die Wahl des Terminus ultramondain rechtfertigte: Von Regionen ausserhalb der sichtbaren Welt ihren Ausgang nehmend, waren die Gravitationskorpuskeln dazu bestimmt (destinés), ihre Wirkung in der sichtbaren Welt zu entfalten. Darin unterschieden sie sich von den Korpuskeln der schweren Materie, was im Begriff ultramondain zum Ausdruck kommen sollte ${ }^{93}$.

Die Textsammlung Physique mécanique enthält eine bemerkenswerte Betrachtung über die Grösse des Kosmos ${ }^{94}$. Unter der Voraussetzung, dass sich die corpuscules ultramondains mit 100000 facher Lichtgeschwindigkeit bewegen und das Alter der Welt 10000 Jahre beträgt, versuchte Lesage, die Grössenordnung der ausserweltlichen Regionen abzuschätzen:

La lumière parcourant plus de 32 millions de lieues en 8 minutes, elle parcourt plus de 20 mille billions en 10 mille ans. Et si les corpuscules gravifiques se meuvent cent mille fois plus vite que la lumière, ils parcourent deux mille trillions de lieues en 10 mille ans. C'est donc à cette distance qu'ont dû être lancés les corpuscules qui causeront la gravité la dix millième année de la création. ${ }^{95}$

Diese beiden Passagen legen ein Modell nahe, dem zufolge Lesage den Kosmos der schweren Materie als begrenzt ansah, wobei die Sichtbarkeit der

92 Die Interpretation Martin Carriers, «diese Korpuskel entstammen einem Vorratsbehältnis jenseits des bewohnten Universums und werden, um der Schöpfung Dauerhaftigkeit zu verleihen, ständig in das Universum hineingeschossen», kann kaum als sachgerecht bezeichnet werden. Vgl. Carrier 1986, 334.

James Evans begnügt sich mit einem lapidaren «called ultramundane because they impinge on us from outside the known universe». Evans 2002, 10.

93 Lesage o.J., 26f.

94 Lesage 1818, 21.

95 Lesage 1818,22f. (Verwendung der Rechenweise: eine Billion gleich eine Million Millionen, eine Trillion gleich eine Million Billionen). Lesages Kalkulation führt auf eine Grössenordnung von $10^{9}$ Lichtjahren für die Grösse des Kosmos, die heutige Schätzung liegt bei $10^{10}$ Lichtjahren. 
schweren Materie gleichzeitig die Grenze des von ihr eingenommenen Raums markierte. Jenseits dieses Kosmos erstreckte sich der unvergleichlich viel grössere «Himmel» der ausserweltlichen Korpuskeln, auf deren Bewegungen und Impulse jede Veränderung im sichtbaren Kosmos letztlich zurückgeführt werden konnte. Sie stellten somit das aktive Prinzip hinter allen Naturvorgängen dar. Offenbar wurde Lesage, wie weiter unten gezeigt wird, auch in diesem Sinne verstanden.

Eine Begründung der Altersangabe von etwa 10000 Jahren gab er nicht. Er konstruierte jedoch aus der Urbewegungsthese einen klassischen Weltseelebeweis auf der Basis eines Mikrokosmos-Makrokosmos-Analogieschlusses, der bei ihm als Bekenntnis zum Theismus interpretiert werden darf:

Il faut avouer que les mouvemens volontaires des hommes sont dus à un être immatèriel ... D'ailleurs, j'entends bien aussi que le mouvement de la matière invisible qui produit les attractions, est dû pareillement à l'action primitive d'un être immatériel..$^{96}$

In seiner theistischen Grundhaltung fand sich Lesage in bestem Einvernehmen mit seinem Genfer Freund Charles Bonnet ${ }^{97}$. Doch fand sie im Unterschied zu Bonnet, der ihr in seiner Naturphilosophie gern breiten Ausdruck verlieh, in Lesages spekulativer Physik selten Niederschlag. Eine Ausnahme bildet eine in den Corrections et additions zum Essai angestellte Untersuchung, wo Lesage die Eigenschaften der corpuscules ultramondains aus Finalursachen ableitete, die ein être ordinateur gesetzt hat: Die «Ökonomie der Natur» erforderte die ausserordentliche Geschwindigkeit und Kleinheit dieser Teilchen. Einerseits kompensierte die hohe Geschwindigkeit den mit der geringen Masse verbundenen Mangel an $\mathrm{Kraft}^{98}$, andererseits war diese Kleinheit erforderlich, um die Zahl ihrer Kollisionen möglichst gering zu halten, sofern dies nicht gar durch das ordnende Wesen überhaupt ausgeschlossen wurde ${ }^{99}$.

Eine weitere wichtige Quelle für die Kosmologie Lesages bildet sein Briefwechsel mit Johann Heinrich Lambert (1728-1777) aus den Jahren 1764 bis $1770^{100}$. Zu dieser Zeit waren dessen Cosmologische Briefe, die zu den grossen kosmologischen Abhandlungen des 18. Jahrhunderts zählen, bereits veröffentlicht ${ }^{101}$. Im Briefwechsel verteidigte Lesage den Begriff ultramondain

96 Lesage 1818, 66. Siehe auch: Lesage o.J., 15. Vgl. hierzu die stoische Argumentation: Cicero, De natura deorum II, 32, und die Ursprünge bei Platon, Phaidros, 245c-e. Zur philosophischen Problematik des Weltseele- und Gottesbegriffs, die Lesage hier komplett übergeht, vgl. Boenke (2005).

97 Prevost 1805, 327 (Briefwechsel mit Bonnet).

98 Lesage o.J., 89. Mit «force» ist hier der Impuls $\mathrm{p}=\mathrm{mv}$ gemeint.

99 Lesage o.J., 89f.

100 Prevost $1805,414-457$.

101 Jackisch 1979, 11. 
gegen eine Kritik, die er Lambert zunächst lediglich unterstellte. Ultramondain implizierte keine räumliche Begrenzung der Welt, erklärte Lesage.

C'est seulement le monde visible, que j'environne de la sorte d'une grande couche de corpuscules, laquelle fait un systême avec lui. Et je remplis tout l'espace de pareils systêmes, qui n'influent les uns sur les autres, que par les corpuscules très-clairsemés ... A peu près comme le firmament visible est parsemés de soleils, qui contribuent peu à s'éclairer les uns des autres. ${ }^{102}$

Es ist also eine Vielzahl voneinander unabhängiger Welten aus schwerer Materie, lediglich durch die allgegenwärtigen Gravitationskorpuskeln verbunden, die Lesage sich vorstellte. Jede dieser Welten besass eine nur begrenzte Dauer wie auch das Universum im ganzen: «Mais je donne un commencement et une fin, tant à chaque systême; qu'à l'univers entier.» ${ }^{103}$

Dass ein derartiges Modell physikalisch auf schwachen Füssen stand, fiel Lambert sofort auf, denn es gab nach dem Forschungsstand keinerlei Anhaltspunkte, dem Bereich der schweren Materie irgendwie eine Grenze zu setzen, genausowenig wie der Wirkung der Gravitation, die ja im Gegenteil den allgemeinen Zusammenhang auch in Lesages Kosmos gerade herstellte. Es wäre nicht bewiesen, so Lambert, dass das Licht sich durch das ganze Universum erstreckte. Ein Blinder z.B. könnte seinem Universum mit der Atmosphäre eine Grenze setzen und dahinter einen unendlichen ausserweltlichen Raum annehmen. Zudem missfiel ihm die Trennung in einen weltlichen und einen ausserweltlichen Bereich, da sie seiner tiefen Überzeugung der Einheit des Kosmos widersprach ${ }^{104}: \ll \ldots$ les causes ordinaires ou mondaines ne peuvent pas désunir ce qui a été uni par le createur.» Man könnte nicht das Unendliche und die absolute Kontinuität, die zwischen den ersten Elementen der Natur herrsche, zerteilen ${ }^{105}$. Auch im Hinblick auf die Frage der Schöpfung und ihres Zeitpunktes vertrat er unter Bezug auf Newton einen gänzlich anderen Standpunkt. Die Frage der Kosmogonie hatte für Lambert wenig Bedeutung. Sein Kosmos war ein statischer, die Welt so erschaffen, dass zu jedem Zeitpunkt ein bestmöglicher Bestand aller ihrer Teile gewährleistet war ${ }^{106}$.

102 Prevost 1805, 420f. (Hervorhebung im Original).

103 Prevost 1805, 420f.

104 Jackisch 1979, 30f., 120.

105 Prevost 1805, 422f.

106 Jackisch 1979, 45. 


\section{Ursprung der Äthertheorie: Kohäsion und chemische Affinität im Essai}

Wenn Pierre Prevost in seinem Buch über den Ursprung der magnetischen Kräfte Lesages Theorie der chemischen Affinität mit Bezug auf den Essai referierte und dabei behauptete, Lesage hätte die chemische Affinität auf die Wirkung eines Äthers zurückgeführt, dann war das nur halb korrekt ${ }^{107}$. Einem weitverbreiteten Trend der Chemie des 18. Jahrhunderts folgend, hatte Lesage in der Schrift von 1758 die Gravitationsanziehung als Grundkraft auch der korpuskularen Ebene behauptet: «Ce Fait, se nomme Attraction en général. Mais il prend le nom de Gravitation en particulier, quand les Corps sont fort éloignés; \& celui de Cohésiom, quand ils se touchent immédiatement.» ${ }^{108}$ Darüber hinaus distanzierte er sich von der Ansicht einiger Newtonianer und Newtons selbst, Erscheinungen der Elastizität, etwa die expansible Wirkung von Luft und Feuer, auf die Wirkung repulsiver Kräfte zurückzuführen ${ }^{109}$.

Im ersten Teil des Essai diskutierte Lesage Kristallisationen und die mit Volumenkontraktion einhergehende Durchmischung von Flüssigkeiten als Musterbeispiele der Kohäsion. Dabei argumentierte er zum einen mit einem modifizierten Gravitationsgesetz, in dem die Massen durch die Dichten der Korpuskeln ersetzt worden waren. ${ }^{110}$ Zum andern behauptete er einen Effekt der Berührung von Oberflächen: Zwei Partikel gleicher oder verschiedener Materie, die in einer Flüssigkeit schwimmen, tendieren dazu, sich über die grösstmögliche Fläche aneinanderzulagern ${ }^{111}$. Neben der Grösse der Berührungsflächen spielte bei diesem Vorgang ihre wechselseitige Passform eine Rolle, z.B. die Ebenheit oder Krümmung. Mechanistische oder dynamische Erklärungen wurden hier nicht vorgeschlagen ${ }^{112}$.

Das preisgekrönte physikomechanische System der chemischen Affinität folgte erst nach Einführung seiner Gravitationstheorie und in Ableitung von ihr. Lesage stellte es unter das traditionsreiche, der Alchemie verpflichtete Motto simile simili gaudet. Dass die chemische Affinität auf der Übereinstimmung der in Verbindungen enthaltenen chemischen Prinzipien beruhte, war im 18. Jahrhundert gängige Ansicht; nur wenige Chemiker, wie Gabriel

107 Prevost 1794, 43.

108 Lesage o.J., 1.

109 Lesage o.J., 6.

110 Lesage o.J., 5.

111 Lesage o.J., 10.

112 Lesage o.J., 12: «Je n'entends ici, par Disposition ou Rapport, que plus ou moins de Densité, plus ou moins de Ressemblance dans la figure des surfaces, \& plus ou moins de Poli dans ces mêmes surfaces.» 
François Venel, der die chemischen Artikel in Diderots Encyclopédie verfasste, vertraten die gegenteilige Position. «Les substances de même nature, s'approchent \& s'attachent mutuëllement, avec plus de force, que les substances de nature diffèrente», behauptete Lesage ${ }^{113}$. Indes fasste er unter dieses Prinzip nicht nur chemische Reaktionen im heutigen Sinne, sondern auch Phänomene wie das Zusammengehen zweier Öl- oder Wassertropfen, die getrennte Kristallisation zweier gelöster Salze oder die Auftrennung der verschiedenen Bestandteile in Milch und Blut. Typisch chemische Reaktionen wie die zwischen Säuren und Basen schienen sich im Gegenteil gerade nicht in dieses Prinzip zu fügen. Dennoch hielt Lesage am Grundsatz der Anziehung des Gleichen fest: Die Lebhaftigkeit derartiger Umsetzungen, so seine Erklärung, beruhte nicht auf einer stärkeren Anziehung, sondern auf einer leichteren gegenseitigen Durchdringung der Partikel auf Grund ihrer unterschiedlichen Grössen, die im Falle gleicher Substanzen natürlich nicht gegeben war $^{114}$.

Lesages Paradigma, an dem er den Mechanismus des simile simili gaudet demonstrierte, bildete das System der Wasser- und Öltropfen. Die Affinität wurde dabei als unmittelbarer Effekt der corpuscules ultramondains interpretiert. Zwei Tropfen Öl oder Wasser wurden demnach durch denselben Mechanismus zusammengetrieben wie zwei Himmelskörper. Der Einfachheit halber setzte er die Anziehungskraft in beiden Fällen als gleich gross an. Sie war identisch mit der Differenz der Kräfte, mit denen ein Öl- bzw. Wassertropfen zum einen von den corpuscules ultramondains in Richtung des zweiten geschoben und zum andern in die entgegengesetzte Richtung von denjenigen corpuscules ultramondains getrieben wurde, die den zweiten Öl- bzw. Wassertropfen passiert hatten ${ }^{115}$. Nun galt es dem Phänomen der Substanzspezifität Rechnung zu tragen. Lesage behauptete, dass die Korpuskeln der auseinandertreibenden Kraft einen Tropfen stärker stiessen, wenn dieser von anderer Beschaffenheit sei als derjenige, den sie passiert haben:

Les Corpuscules qui ont traversé les Pores d'un Corps; passent plus aisément par les Pores d'un second Corps, lorsque ceux-ci sont de même sorte que ceux-là, que lorsqu'ils sont de differente espèce. C'est à quoi nous mène nècessairement, la superiorité de l'Attraction mutuëlle des Corps de nature differente. ${ }^{116}$

Die Ursache für dieses unterschiedliche Durchdringungsverhalten der auseinandertreibenden Gravitationsatome suchte Lesage in der Porenstruktur

113 Lesage o.J., 30.

114 Lesage o.J., 33.

115 Lesage o.J., 36.

116 Lesage o.J., 37. 
der schweren Materie. Dabei gelangte er schliesslich dazu, die Abfangrate für Gravitationskorpuskeln als Unterscheidungskriterium für die chemische Beschaffenheit der schweren Korpuskeln zu benutzen.

Im folgenden soll seine Argumentation, da sie das theoretische Kernstück des Essai darstellt, kurz referiert werden. Betrachtet wird ein System zweier Wassertropfen. Die Abfangrate eines Wassertropfens setzte er - vollkommen willkürlich - mit $1 / 7$ an. Ein Wassertropfen wird also durch die Impulse des siebten Teils eines Gravitationsstroms, der ihn von der dem zweiten Wassertropfen entgegengesetzten Richtung passiert, zu diesem zweiten Wassertropfen hingetrieben. Er wird zweitens von 1/7 des Gravitationsstroms aus der Richtung des zweiten Wassertropfens von diesem weggetrieben. Nun hat dieser zweite Gravitationsstrom bereits einen Wassertropfen passiert und besitzt daher nur $6 / 7$ der Stärke des entgegengesetzten Stroms. Die Kraft, mit der der erste Tropfen also auf den zweiten zudriftet, ist gleich der Differenz der beiden Ströme, das ist $1 / 49$ eines der ursprünglichen Ströme. Dasselbe gilt für den zweiten Tropfen ${ }^{117}$. Die Anziehungen zwischen zwei Öl- oder einem Wasser- und einem Öltropfen wären konsequenterweise nach demselben Schema zu berechnen gewesen. Lesage setzte die Abfangrate eines Öltropfens mit $1 / 5$ an. Die analoge Rechnung würde nun für die anziehende Kraft zwischen einem Öl- und einem Wassertropfen je einen Wert von $1 / 35$ ergeben. Da dieses Ergebnis im Widerspruch zur These der stärkeren Anziehung des Gleichen stand, führte Lesage eine Hilfskonstruktion ein, die als Keim seiner späteren Äthertheorie angesehen werden muss. Ihre Einführung bedeutet gleichzeitig ein Scheitern seines Versuchs, die Wirkungen der chemischen Affinität allein auf Korpuskelform und Gravitationskraft zurückzuführen: Lesage teilte den Korpuskelstrom in eine gröbere und eine feinere Hälfte und setzte für beide Teile unterschiedliche Abfangraten an, $1 / 5$ für die gröbere und $1 / 35$ für die feinere Hälfte:

Supposons:... que chaque goutte d'Huile isolée, arrête la cinquiéme partie de la moitié la plus grossière du Courant qui y aborde; \& que ces mêmes gouttes d'Huiles, arrêtent la trentecinquiéme partie de la moitié la plus subtile. ${ }^{118}$

Unter dieser Voraussetzung ermittelte er für die Anziehung zwischen Öl- und Wassertropfen einen Wert von $4 / 245$ des Partikelstroms, womit die stärkere Anziehung des Gleichen bewiesen war. Ein solcher Strom aus gröberen und subtileren Partikeln entsprach im Prinzip nicht mehr dem ursprünglichen Begriff der corpuscules ultramondains, da er eine Substanzspezifität der Gravitationskraft implizierte. Lesage behielt dennoch seine Terminologie bei. Nun

117 Lesage o.J., $44 f .:[1 / 7-1 / 7 \times 6 / 7]=[7 / 49-6 / 49]=1 / 49$.

118 Lesage o.J., 44. 
sollte - im Widerspruch zu den vorherigen Überlegungen - der Anteil an gröberen Partikeln relativ gering sein: «Mais alors, il faut, que la portion subtile des Courans Ultramondains, soit beaucoup plus abondante que leur portion grossière.» ${ }^{119}$

Damit war sein preisgekrönter Versuch, die chemischen Affinitäten als unmittelbare Wirkungen der Gravitation zu deuten, fehlgeschlagen. In den später verfassten Schriften nahm er diesen theoretischen Ansatz folgerichtig nicht mehr auf. Die chemische Affinität wurde jetzt auf die Wirkung sekundärer Fluida zurückgeführt, subtile Partikelströme, die ihrerseits von den corpuscules ultramondains bewegt wurden ${ }^{120}$. Diese sekundären Fluida bezeichnete Lesage als Äther.

\section{Äther und elastische Fluida}

In der späteren Äthertheorie der chemischen Affinität entwickelte Lesage denjenigen Aspekt der Theorie von 1758 weiter, der die Reduktion der chemischen Anziehungen auf Gravitationskräfte zu Fall gebracht hatte. Ihre Substanzspezifität liess sich durch die Einführung eines heterogenen, subtilen Fluidums, das sich aus verschieden grossen Korpuskeln zusammensetzte, die nicht mit den corpuscules ultramondains identisch waren, sehr viel besser deuten. Ein derartiges Fluidum bezeichnete Lesage als Äther. Es bestand aus gröberen als den Gravitationsatomen, wurde aber von diesen unmittelbar und permanent in Bewegung gehalten. Eine prägnante Beschreibung des Lesageschen Äthers und seiner Unterschiede zu den corpuscules ultramondains hat Prevost in seinem Ursprung der magnetischen Kräfte gegeben:

Seine [des Äthers] Agitation entspringt aus der Ungleichheit der entgegengesetzten Ströme, welche auf die Grundmassen stoßen. Dieser, ohne Unterlaß bewegte, Aether bringt folglich, ohne Unterlaß, sehr schnelle Ströme hervor. Diese Ströme werden unterbrochen und dauren nicht immer fort, wie die der schwermachenden Theilchen; aber es entstehen unaufhörlich neue. Man kann also auf die Bewegung des Aethers beynahe dieselben Vorstellungen anwenden, welche man sich von den schwermachenden Körpertheilchen gemacht hat. Diese beiden Flüssigkeiten sind aber doch in einer andern Rücksicht wesentlich von einander verschieden. Die eine, nämlich die schwermachende Flüssigkeit, bestehet aus Elementen, welche der Masse nach nicht merklich von einander verschieden sind; die andere, der Aether, ist im entgegengesetzten Falle: seine Elemente sind der Masse nach merklich von einander verschieden, und also auch dem Volum nach; denn alle diese kleinen Körperchen sind hart, einfach und elementarisch. ${ }^{121}$

119 Lesage o.J., 47.

120 Prevost 1818, 68, Fussnote.

121 Prevost 1794, 48f. 
Damit war ein Fluidum konzipiert, das sich von den Gravitationsatomen beachtlich unterschied. Die Bewegungen der Ätheratome waren, obwohl durch die corpuscules ultramondains verursacht, gänzlich anderer Natur. Es konnten, so die Überlegung, in einem gegebenen Zeitmoment die einem Ätheratom versetzten Gravitationsimpulse nicht von allen Seiten her gleich sein. Damit ergab sich eine Asymmetrie der Verteilung von Impulsen, aus der eine unregelmässige Hin-und-her-Bewegung resultierte. Lesage verglich sie mit einer Schwingung und bezeichnete sie als agitation ${ }^{122}$. Diese Art der Bewegung bedingte die nur geringe Reichweite der chemischen Kräfte und beruhte nach Lesages korpuskularmechanischer Interpretation auf der vollständig konvexen Form der Ätheratome. Die Impulse der Gravitationsatome auf die völlig gerundeten Oberflächen der Ätheratome bildeten die sogenannte erste Bewegungsursache.

... on peut concevoir les particules comme entièrement convexes et qui ne sont agités que par la première cause que nous avons remarquée, c'est-à-dire, parce que les corpuscules ultramondains ne les atteignent pas précisement en même temps. Ce ne peut être, nous avons dit, que des fluides très-subtils; on pourroit, pour les distinguer des autres, les nommer éthers. ${ }^{123}$

Ein weiterer fundamentaler Unterschied sollte die Affinitätskräfte gegenüber der Gravitation auszeichnen: die Abhängigkeit von der Körperoberfläche.

Dieser Äther ist mit den Flüssigkeiten, welche in unsere Sinne fallen, verglichen, sehr subtil; aber in Vergleichung mit dem schwermachenden Fluidum sehr grob. Seine Wirkung auf die Partikeln, die ihn aufhalten, ist also nicht der Masse dieser Partikeln, sondern vielmehr ihren Flächen proportional. ${ }^{124}$

Gerade die im Newtonschen Gravitationsgesetz formulierte Massenabhängigkeit der Schwerkraft hatte Lesages Theorie der pushing gravity beträchtliche Schwierigkeiten bereitet und ihn zum Konzept der extrem durchlässigen Materie gezwungen. Auf korpuskulartheoretischer Ebene konnte er einer gleichsam natürlichen Konsequenz der Impulstheorie nachgeben. Für den dichteren Äther war die Wahrscheinlichkeit, sich in den Poren und Gängen der chemischen Substanzen zu verfangen, sehr viel höher ${ }^{125}$.

Angesichts der Gesamtheit an Neuerungen und Präzisierungen des Ätherbegriffs gegenüber dem Ansatz im Essai erstaunt es um so mehr, dass Lesages spätere Texte über die Mechanismen chemischer Prozesse die Überlegungen und Rechenbeispiele des Essai genau wiederholen. Lediglich die Terminologie hat sich gewandelt. An die Stelle der corpuscules ultramon-

122 Prevost 1794, 38; Lesage 1818, 128.

123 Lesage 1818, 139 (Hervorhebung im Original).

124 Prevost 1794, 52f.

125 Prevost 1794, 53. 
dains trat nun der Äther ${ }^{126}$. Schliesslich stellte Lesage am Ende seiner Untersuchung über den Mechanismus der Anziehung des Gleichen fest, dass allein die Verhältnisse der Durchlässigkeiten chemischer Körper für die verschiedenen Atome des Ätherstroms dieses angebliche Phänomen verursachen: «... il suffit que ces rapports ne soient pas égaux, pour que la tendance mutuelle des corps, d'une même espèce soit supérieure à la tendance mutuelle de ceux de différente espèce.» ${ }^{127}$

Vom Äther zu unterscheiden war eine andere Kategorie subtiler Materie, die elastischen oder expansiblen Fluida. Hierzu zählten das Licht, das Feuer, die Elektrizität und der Magnetismus. Bei Pierre Prevost gewannen die elastischen Fluida vor allem im Hinblick auf die Theorie der Gase und der Wärme an Bedeutung ${ }^{128}$. Für die elastischen Fluida galt ein anderer Bewegungsmechanismus, der durch die Form ihrer Korpuskeln bedingt war. Sie besassen Einwölbungen, Konkavitäten, in denen sich die corpuscules ultramondains verfingen und herumrollten. Besass eine Partikel eine Konkavität, so wirkten die Impulse also nicht gleichmässig von allen Seiten, sondern asymmetrisch, so dass sich eine bevorzugte Bewegungsrichtung ausbildete. Das in der Konkavität rollende Gravitationsatom fungierte dabei als eine Art Motor. Den Gravitationsströmen ausgesetzt, wird sich eine Partikel in die Richtung bewegen, die ihrer Konkavität sozusagen gegenüberliegt ${ }^{129}$. Lesage verglich diese Bewegung mit der eines Schiffes, die Teilchen ausgestattet mit Bug und Heck ${ }^{130}$. Nach diesem Mechanismus, der sogenannten zweiten Bewegungsursache, sollte sich auch das Licht bewegen. Die in der Konkavität der Lichtpartikeln rollenden Gravitationsatome verursachten eine geradlinige Ausbreitung. Allerdings war das Licht das einzige der elastischen Fluida, dessen Geschwindigkeit gemessen werden konnte ${ }^{131}$.

Die Theorie der elastischen Fluida gehört einer späteren Phase des Lesageschen Denkens an. Noch im Essai hatte er einen ausdrücklichen Verzicht auf die Beschäftigung mit dieser Fragestellung erklärt und sich mit der lapidaren Behauptung begnügt, dass es wahrscheinlich keines Rekurses auf repulsive Kräfte bedarf, um dieses Problem zu lösen. Diesbezügliche Überlegungen bei Newton und einigen seiner Anhänger hatte er ausdrücklich zurückgewiesen ${ }^{132}$.

126 Lesage 1818, 156.

127 Lesage 1818, 166.

128 Prevost 1828.

129 Prevost 1794, 40f.

130 Lesage 1818,137f.

131 Lesage 1818, 144.

132 Lesage o.J., 2, 6f. 


\section{Literaturverzeichnis}

Aronson, Samuel, "The Gravitational Theory of Georges-Louis Lesage”, Natural Philosopher 3 (1964) 53-74

Boenke, Michaela, «Wirbeltheorien», in: Joachim Ritter (Hrsg.), Historisches Wörterbuch der Philosophie (Basel 2004)

- «Gott und seine Mitregenten. Theologische, stoische und platonische Elemente in der Naturtheorie Newtons», in: Thomas Leinkauf (Hrsg.), Der Naturbegriff in der Frühen Neuzeit (Tübingen 2005)

Böhme, Gernot/Hartmut Böhme, Feuer, Wasser, Erde, Luft. Eine Kulturgeschichte der Elemente (München 1996)

Bopp, Karl, «Die wiedergefundene Abhandlung von Fatio de Duillier: De la Cause de la Pesanteur», in: Karl Bopp, Drei Untersuchungen zur Geschichte der Mathematik, Schriften der Straßburger Wissenschaftlichen Gesellschaft in Heidelberg N.F. 10. Heft (Berlin/Leipzig 1929)

Carrier, Martin, «Die begriffliche Entwicklung der Affinitätstheorie im 18. Jahrhundert», Archive for the History of Exact Sciences 36 (1986) 327-389

Duncan, Alistair, Law and Order in Eighteenth-Century Chemistry (Oxford 1996)

Edwards, Matthew (ed.), Pushing Gravity: new perspectives on Le Sage's theory of gravitation (Montreal 2002)

Euler, Leonhard, Briefe an eine deutsche Prinzessin über verschiedene Gegenstände der Physik und Philosophie (Leipzig 1769-1773, Nachdruck Braunschweig 1986)

Evans, James, «Gravity in the Century of Light», in: Edwards (2002) 9-40

Gagnebin, Bernard, «De la cause de la pesanteur. Mémoire de Nicolas Fatio de Duillier», Notes and Records of the Royal Society of London 6 (1949) 105-160

Isenkrahe, C., «Euler's Theorie der Gravitation», Zeitschrift für Mathematik und Physik. Historisch-literarische Abtheilung XXVI (1881) 1-19

Jackisch, Gerhard, Johann Heinrich Lamberts «Cosmologische Briefe» mit Beiträgen zur Frühgeschichte der Kosmologie (Berlin 1979)

Jürss, Fritz/Reimar Müller/Ernst Günther Schmidt (Hrsg.), Griechische Atomisten. Texte und Kommentare zum materialistischen Denken der Antike (Berlin [West] 1984)

Kleinert, Andreas (éd.), «Correspondance d'Euler avec George-Louis Lesage», in: Leonhard Euler, Opera omnia, Ser. IV A, Vol. 7 (im Druck)

Kurdzialek, M., «Äther», in: Joachim Ritter (Hrsg.), Historisches Wörterbuch der Philosophie (Basel/Stuttgart 1971) Bd. 1, 599-601

Laudan, Laurens, «George-Louis Lesage: A Case Study in the Interaction of Physics and Philosophy», Akten des II. Internationalen Leibniz-Kongresses, Hannover 17.-22.7.1972, Bd. II Wissenschaftstheorie und Wissenschaftsgeschichte; Studia Leibnitiana Supplementa VIII (Wiesbaden 1974) 241-252

- «The medium and its message: a study of some philosophical controversies about ether», in: G. N. Cantor/M. J. S. Hodge (eds), Conceptions of ether. Studies in the history of ether theories (Cambridge 1981) 157-185

Leibniz, Gottfried Wilhelm, «Streitschriften zwischen Leibniz und Clarke», in: Gottfried Wilhelm Leibniz, Hauptschriften zur Grundlegung der Philosophie, hrsg. von Ernst Cassirer, Bd. 1 (Hamburg 1904, Nachdruck 1966) 81-182

Lesage, George-Louis, Essai de Chymie méchanique (o.J.)

- «Lucrèce Neutonien», dans: Prevost (1805) 561-604

- «Exposition du système des corpuscules ultramondains», dans: Prevost (1818) 3-86

- «Des fluides élastiques ou expansifs», dans: Prevost (1818) 123-151

- «Application des théories précédentes a certaines affinités», dans: Prevost (1818) 152186

Lukrez, Vom Wesen des Weltalls (De natura rerum), übersetzt von Dietrich Ebener (Leipzig 1989)

Nobis, H. M., «Äther», in: Robert Auty (Hrsg.), Lexikon des Mittelalters, Bd. 1 (München 2002) $1164 f$.

Pohlenz, Max (Hrsg.), Stoa und Stoiker. Die Gründer, Panaitios, Poseidonios (Zürich $\left.{ }^{2} 1964\right)$ 
Prevost, Pierre, Vom Ursprunge der magnetischen Kräfte (Halle 1794)

- (éd.), Notice de la vie et des écrits de George-Louis Lesage de Genève (Genève 1805)

- (éd.), Deux traités de physique mécanique (Genève/Paris 1818)

- Mémoire sur la constitution des fluides élastiques (Genève 1828)

Rosenberger, Ferdinand, Die Geschichte der Physik, Bd. III (Braunschweig 1887-1890)

Stosz, Wilhelm, Le Sage als Vorkämpfer der Atomistik (Dissertation, Halle 1884)

Thackray, Arnold, «Matter in a Nut-Shell: Newton's Opticks and Eighteenth-Century Chemistry», Ambix 15 (1968) 29-53

- Atoms and Powers. An Essay on Newtonian Matter-Theory and the Development of Chemistry (London 1970)

Weiss, Burghard, Zwischen Physikotheologie und Positivismus. Pierre Prevost (1751-1839) und die korpuskularkinetische Physik der Genfer Schule (Frankfurt am Main 1988)

Zehe, Horst, Die Gravitationstheorie des Nicolas Fatio de Duillier (Hildesheim 1980) 